\title{
Iron deposition in rabbit cerebellum after exposure to generated and mobile GSM electromagnetic fields
}

\author{
Kopani $\mathrm{M}^{1}$, Filova B ${ }^{1}$, Sevcik $\mathrm{P}^{1}$, Kosnac $\mathrm{D}^{1}$, Misek $\mathrm{J}^{2}$, Polak $\mathrm{S}^{3}$, Kohan $\mathrm{M}^{2}$, Major $\mathrm{J}^{4}$, \\ Zdimalova $\mathrm{M}^{4}$, Jakus $\mathrm{J}^{2}$ \\ Institute of Medical Physics, Biophysics, Informatics and Telemedicine, Faculty of Medicine in Bratislava, \\ Comenius University, Slovakia. martin.kopani@fmed.uniba.sk
}

\begin{abstract}
BACKGROUND: Mobile phone application may cause structural, functional changes and accumulation of toxic elements in brain.

OBJECTIVES: The aim of this study was to investigate iron accumulation in rabbit cerebellum after exposure to RF EMF with light and scanning electron microscopy.

MATERIALS AND METHODS: Histochemical analysis of iron distribution by light and electron microscopy with energy-dispersive microanalysis was used.

RESULTS: Light microscopy revealed dystrophic changes of Purkinje cells in irradiated groups and iron deposits located in various parts of cerebellum. Deposits consists of $\mathrm{C}, \mathrm{O}, \mathrm{Na}, \mathrm{Mg}, \mathrm{Al}, \mathrm{Si}, \mathrm{P}, \mathrm{S}, \mathrm{Cl}, \mathrm{Ca}$ and $\mathrm{Fe}$. CONCLUSION: Our experiment revealed structural changes of Purkinje cells and iron and aluminium accumulations in stratum granulosum of rabbit's cerebellum after exposure to RF EMF (Fig. 6, Ref. 33). Text in PDF www.elis.sk.

KEY WORDS: brain, radiofrequency electromagnetic field, electron microscopy.
\end{abstract}

\section{Introduction}

During mobile phone usage, human body is exposed to radiofrequency electromagnetic field (RF EMF). Evidences that exposure to RF EMF might affect people's health exist $(1,2)$. RF EMF is classified as possibly carcinogenic to humans (Group 2B carcinogens) by International Agency for Research on Cancer (IARC) (3). Many studies revealed adverse effects of RF EMF on the cardiovascular, immune and cerebrospinal systems and production of various enzymes and proteins (4-8). Mobile phone in a calling mode is placed in a close proximity to a user's head that increases absorption of energy by brain (9). This could affect and alter physiological brain activity and the function of the central

${ }^{1}$ Institute of Medical Physics, Biophysics, Informatics and Telemedicine, Faculty of Medicine in Bratislava, Comenius University, Slovakia, ${ }^{2}$ Department of Medical Biophysics, Jessenius Faculty of Medicine in Martin, Comenius University in Bratislava, Slovakia, ${ }^{3}$ Institute of Histology and Embryology, Faculty of Medicine in Bratislava, Comenius University, Slovakia, and ${ }^{4}$ Department of Mathematics and Descriptive Geometry, Faculty of Civil Engineering, Slovak University of Technology in Bratislava, Slovakia

Address for correspondence: Martin Kopani, Institute of Medical Physics, Biophysics, Informatics and Telemedicine, Faculty of Medicine in Bratislava, Comenius University, Slovakia.

Phone: +421.2 .59357530$

Acknowledgements: This work was supported by Slovak Agency for Research and Development - the project No. APVV-0189-11(prof. Jakuš) and APVV-16-0039. nervous system - CNS (10-12). However, the precise mechanism of RF EMF interaction with cells and tissues is still unknown. Consequences of the interactions are possibly the changes in neurotransmitter systems, hormone production and/or blood-brain barrier (BBB) integrity (13). BBB impedes the influx of toxic molecules from blood into the brain. However, this impairment of BBB by exposition to RF EMF is still controversial $(14,15)$. Higher permeability of BBB causes that some substances, like toxins and other harmful material, leak out of a bloodstream into the brain cells and tissues.

Iron is a natural chemical element of a human body participating in plenty of biochemical reactions responsible for myelin production, growth and development. It is a part of some enzymes and proteins and may play an important role in the development of neurodegenerative diseases and the aging processes (16). It is one of the elements, whose abnormal quantity induces the oxidative stress. Iron can be found in human blood, but can also accumulate in the brain due to BBB impairment.

The aim of this study was to investigate iron accumulation in rabbit cerebellum after exposure to RF EMF with light and scanning electron microscopy.

\section{Material and methods}

All procedures were conducted in accordance with the Declaration of Helsinky and with the laws, rules, and regulations of the Slovak Republic, and Comenius University Bratislava, taking into account the Directive 2010/63/EU of the European Parliament. 


\section{5-579}

Permissions were covered by the Slovak Agency for Research and Development - the project No. APVV-0189-11 (prof. Jakus). Experiments were performed on 32 (30 males and 2 females; $3.89 \pm 0.5 \mathrm{~kg}$ ) spontaneously breathing New Zealand White Rabbits. Rabbits were initiated to anaesthesia by combination of tiletaminum and zolazepamum (Zoletil 100, Virbac, France) in dose $30 \mathrm{mg} / \mathrm{kg}$ i.m. Recurrent supplemental anaesthesia was $\alpha$-Chloralose (Sigma-Aldrich, USA) in dose $60 \mathrm{mg} /$ $\mathrm{kg}$ i.p. as needed. The trachea and femoral vein were cannulated. The animals were allowed to breathe spontaneously, while endtidal $\mathrm{CO}_{2}$, respiration rate and body temperature were monitored continuously. Animals did not suffer from any disease. Each rabbit was individually placed into the Faraday exposure cage. During an exposure, the background extremely low frequency magnetic flux density did not exceed $80 \mathrm{nT}$ for the frequency band of $5 \mathrm{~Hz}-100 \mathrm{kHz}$ as measured by Narda EHP50-D (Narda Safety Test Solution, Pfullingen, Germany). The horizontal and vertical components of static magnetic field were $49 \pm 4 \mu \mathrm{T}$ and $80 \pm 3$ $\mu \mathrm{T}$ respectively. Rabbit's head was placed $5 \mathrm{~cm}$ from the loop antenna (17). The occipital and parietal brain regions were primarily exposed during $150 \mathrm{~min}$.

The rabbits were divided into four groups:

1st group exposed by real telecommunication GSM (Global System for Mobile Communications) signal (10 individuals) characterized by frequency band $1805-1870 \mathrm{MHz}$, corresponding to Slovak mobile providers downlink. The signal was calibrated to intensity of electric field (E) $300(\mathrm{~V} / \mathrm{m}$ ) measured by broadband meter Narda NBM550 (Narda Safety Test Solutions, Pfullingen, Germany).

2nd group was exposed by generated electromagnetic field (7 individuals). The signal was pulse modulated with duty cycle 50 $\%$ under carrier frequency $1788 \mathrm{MHz}$ generated by functional generator Agilent N9310A (Agilent Technologies, Santa Clara, USA) and amplified by laboratory $5 \mathrm{~W}$ amplifier AR 5S1G4 (Amplifier Research, Souderton, USA). E field was calibrated to 160 (V/m).

3rd group was exposed to combination of both the real and generated signals (6 individuals), thus rabbit's head was exposed to combination of the groups No. 1 and 2.

4th group served as control (9 individuals). Animals in sham group were held always at the same conditions as animals in groups 1,2 or 3 . Thus, they were affected with same anaesthetics and kept within the same exposure unit during the same time period, however, without RF exposure.

At the end of the trial, the animals were sacrificed by anaesthetic agent Sodium pentobarbital (Morbital, Biowet, Poland; 160 $\mathrm{mg} / \mathrm{kg}$ i.v.) followed by dose of $5 \mathrm{ml}$ Potassium chloride ( $\mathrm{KCl})$ i.v. The brain was gently removed from the skull and placed in $37 \%$ formaldehyde. After the fixation, the brain tissue was dehydrated in an ascending series of alcohol, cleared with xylene and embedded in paraffin wax. Serially coronal sections were cut on the rotating microtome ( $5 \mu \mathrm{m}$ thick) and mounted on silanized slides. Afterward, slides were stained with haematoxylin and eosin according to standard protocol. For iron visualization, the Prussian blue reaction was used. The tissue sections were incubated in freshly prepared $4 \%$ potassium ferrocyanide (EC237-722-2, Sig-
maAldrich LLC, USA) and $4 \%$ hydrochloride acid for 20 minutes and then rinsed in distilled water. Slides were counterstained with nuclear fast red (N 3020, SigmaAldrich LLC, USA) for 5 minutes, dehydrated in ethanol, cleared with xylene and coverslipped with Entellane (107960, Merck Millipore, Darmstadt, Germany). Neuropathology was assessed with an examiner who was blinded to the experimental procedure. During the sample preparation, special attention was paid to avoid manipulations with instruments containing iron.

Number of iron complexes, taken by light microscope after the Perl's reaction in two image regions with image processing using graph theory algorithms, was obtained. The first region represents the layer of stratum granulosum in vicinity of Purkinje cells and the second region of the same area corresponds to the remote layer of stratum granulosum. Software counted the number of pixels ratio representing iron complexes to the number of pixels representing background in two regions.

Samples for SEM + EDX investigation were mounted on carbon block and covered by carbon layer with $30 \mathrm{~nm}$ thickness. SEM analysis was performed by scanning electron microscope EVO LS 15 (ZEISS) with an accelerating voltage of $20 \mathrm{kV}$. Simultaneous EDX line analysis was performed by AMETEK (EDAX) EDS Element Silicon Drift Detector. The time period of spectrum collection was $200 \mathrm{~s}$ with the energy range 0.160 to $10 \mathrm{keV}$.

\section{Results}

\section{Light microscopy}

We found dystrophic changes of Purkinje cells in all irradiated groups. Their nucleus membranes were thickened and some Purkinje cells were pyknotic. All investigated samples revealed iron deposits located in various parts of cerebellum. In control group, no deposition of iron was observed.

After exposure to real GSM RF EMF signal (group No. 1), iron depositions appeared in samples mainly in stratum granulosum of rabbit's cerebellum (Fig. 2). The size of deposits was around 5 $\mu \mathrm{m}$ in diameter (Fig. 1).

Samples after exposition to RF EMF from generator (group No. 2) revealed the iron depositions of spherical shape in stratum granulosum of rabbit's cerebellum in a close vicinity of Purkinje cells. The size of deposits was around $20 \mu \mathrm{m}$ in diameter (Fig. 2).

Samples after exposure to combined real and generated signals (group No. 3), revealed iron depositions mainly in stratum granulosum of rabbit's cerebellum (Fig. 3). The size of deposits was approximately $5 \mu \mathrm{m}$ in diameter.

Green hue outlined iron complexes in stratum granulosum of rabbit's cerebellum after exposure to real GSM RF EMF signal (group No. 1). It reveals higher concentration of iron deposits in the layer closer to the Purkinje cells (55\%, upper part of the figure) than the remote layer ( $45 \%$, Fig. 4$)$.

\section{Electron microscopy and EDX microanalysis}

Samples after exposure to real GSM signal (group No. 1), revealed smaller iron deposits in stratum granulosum of rabbit's cerebellum compared to the signal from generator (group No. 2). 


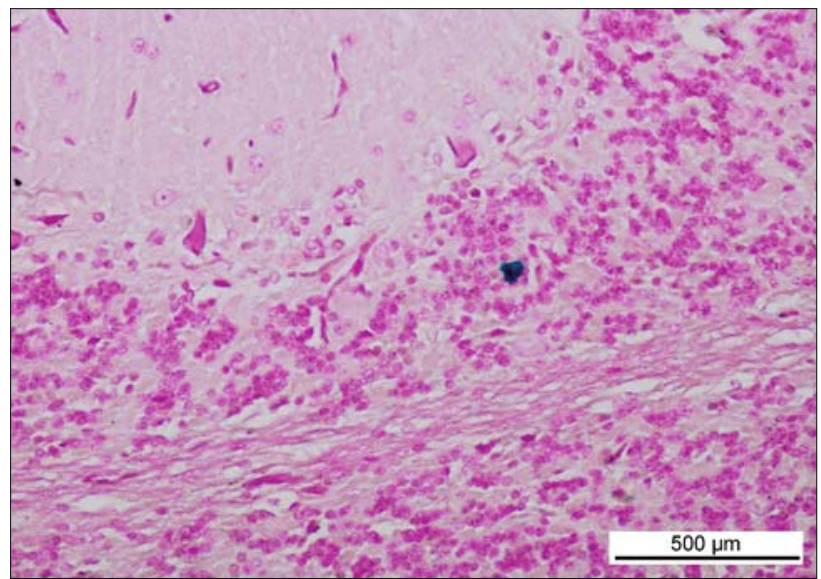

Fig. 1. Rabbit's cerebellum. Blue dye iron deposits in stratum granulosum in vicinity of Purkinje cells after exposition to real GSM RF EMF signal. Perl's reaction. Light microscopy, scale bar $=100 \mu \mathrm{m}$.

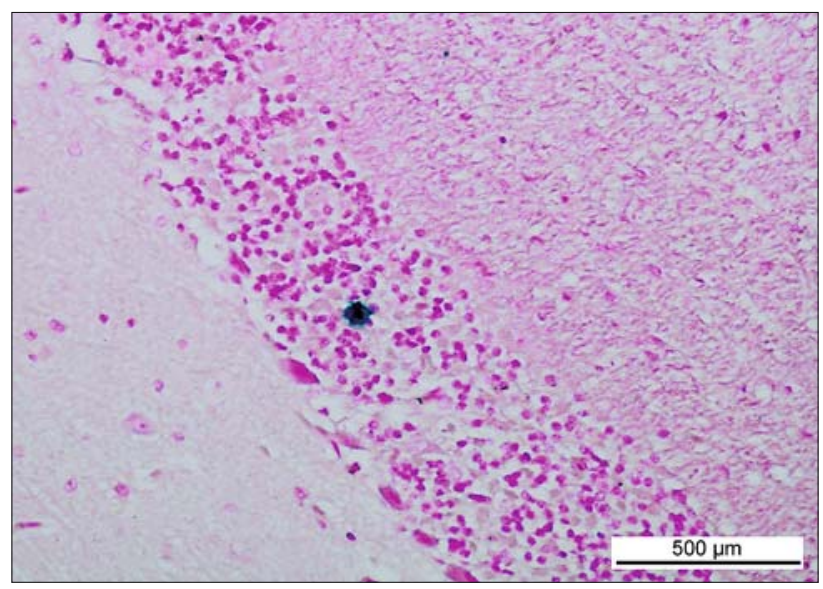

Fig. 2. Rabbit's cerebellum. Blue dye deposits of iron in stratum granulosum in vicinity of Purkinje cells after exposition to RF EMF from generator. Perl's reaction. Light microscopy, scale bar $=100 \mu \mathrm{m}$.

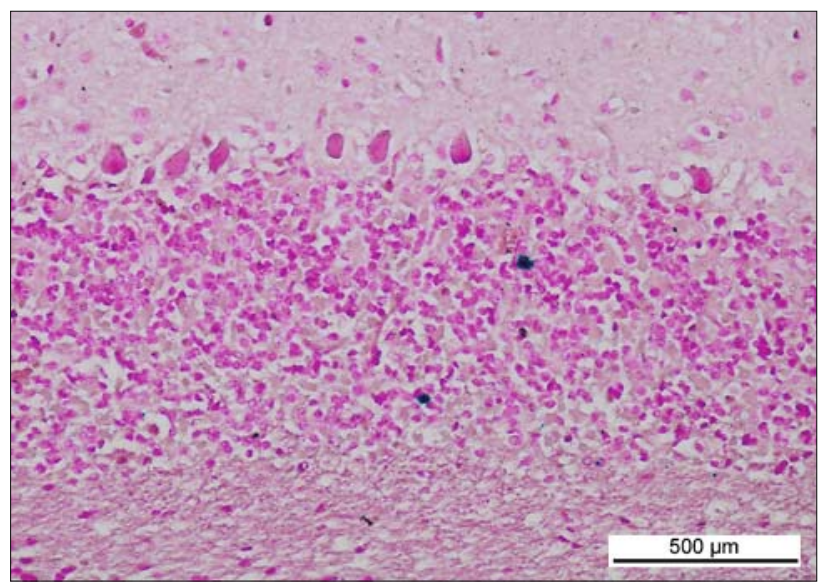

Fig. 3. Rabbit's cerebellum. Blue dye deposits of iron in stratum granulosum in a vicinity of Purkinje cells after exposure to combined real and generated signals. Perl's reaction. Light microscopy, scale bar $=100 \mu \mathrm{m}$.

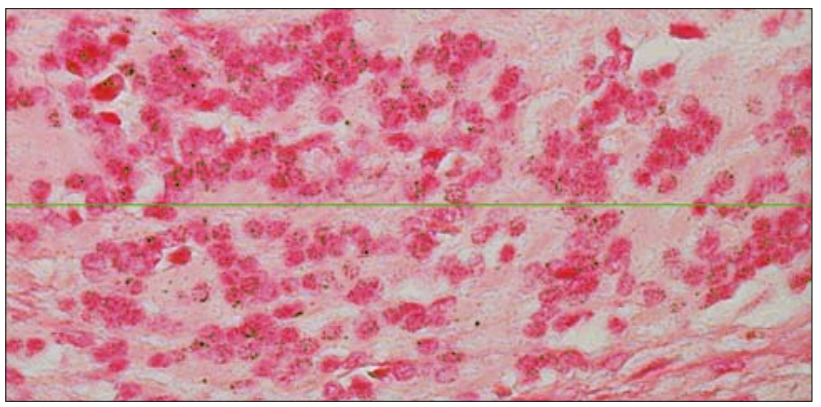

Fig. 4. Rabbit's cerebellum. Green dye deposits of iron in stratum granulosum in vicinity of Purkinje cells (upper part of the figure) after exposition to real GSM RF EMF. Image processing, light microscopy.

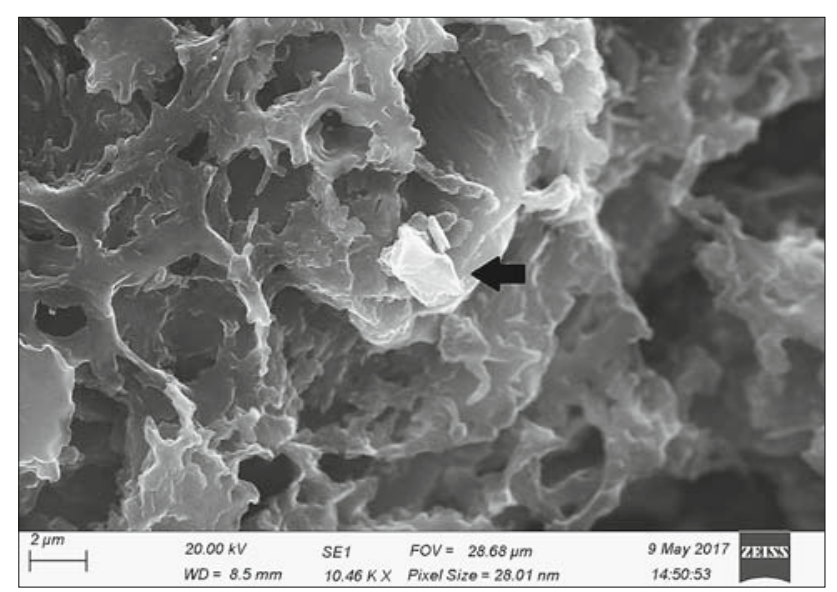

Fig. 5. Rabbit's cerebellum. Iron deposits in stratum granulosum of cerebellum after exposure to real RF signal. Electron microscopy, scale bar $=2 \mu \mathrm{m}$.

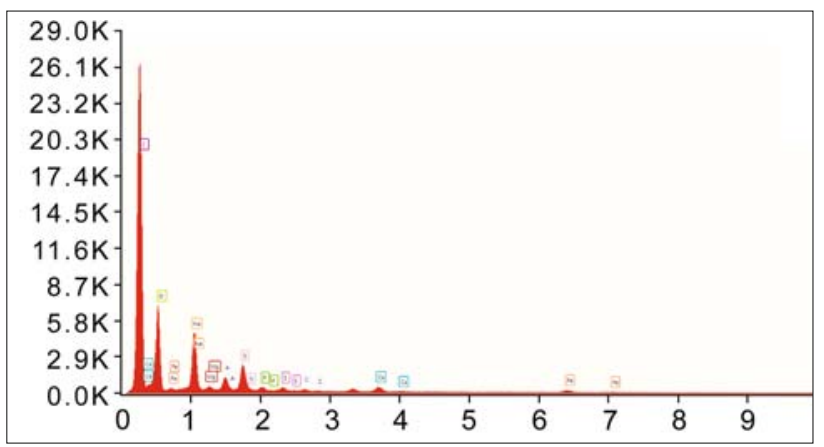

Fig. 6. Rabbit's cerebellum. Iron particle in stratum granulosum of cerebellum after exposure to real RF signal. Particle consists of $C, O$, $\mathrm{Na}, \mathrm{Mg}, \mathrm{Al}, \mathrm{Si}, \mathrm{P}, \mathrm{S}, \mathrm{Cl}, \mathrm{Ca}$ and Fe. SEM-EDX, $20 \mathrm{kV}$.

Deposits were located entirely in an extracellular space and/or on the surface of the cells (Fig. 5).

EDX microanalysis of particles was performed on Fig. 6. It showed isolated, irregular particles of multi-elemental composition in a vicinity of Purkinje cells in the stratum granulosum. Particles consisted of $\mathrm{C}, \mathrm{O}, \mathrm{Na}, \mathrm{Mg}, \mathrm{Al}, \mathrm{Si}, \mathrm{P}, \mathrm{S}, \mathrm{Cl}, \mathrm{Ca}$ and $\mathrm{Fe}$. Other particles of similar size and morphology with content of $\mathrm{C}, \mathrm{O}, \mathrm{Na}$, $\mathrm{Al}, \mathrm{Si}$ and $\mathrm{Ca}$ were also detected (not shown). 


\section{Discussion}

The exact mechanism of RF EMF exposure and coupling with cells and tissues is still unknown. There are evidences that RF EMF can cause structural changes of cerebellum. Rosli and Teoh (18) observed that low frequency EMF induced reduction of Purkinje cells and thinning of the granular layer of the cerebellar cortex in adult mice. Our results using a high frequency EMF revealed dystrophic changes of Purkinje cells that can lead to the reduction of these cells. Light and electron microscopy confirmed moderate accumulation of iron in rabbit's cerebellum after the exposure to RF EMF in the layer closer to the Purkinje cells. Reduction of Purkinje cells and thinning of the granular layer of the cerebellar cortex could be caused by iron inducing formation of reactive species (oxygen and nitrogen), resulting in formation of various complexes and impairment of Purkinje cells and granular layer. Burlaka et al (19) observed formation of iron-nitrosyl complexes of nitric oxide radicals with FeS proteins (NO-FeS complexes) on electron transport chain of mitochondria after exposure to RF EMF. SEM-EDX microanalysis confirms iron-rich particles with oxygen and sulphur, although without nitrogen. This observation can indicate formation of not only iron-oxides minerals, but also very complicated complexes. Apart from above mentioned chemical elements, the $\mathrm{Al}, \mathrm{Si}$ and $\mathrm{Ca}$ particles were detected, too. These findings indicate deposition of other toxic elements.

After the exposure to RF EMF, we did not find any iron accumulation formation in another brain location. Naturally, iron, as the most abundant transition metal in the brain and blood, can be regularly found in brain tissue, although in less extent. The mechanism of short-lasting RF EMF exposure on cerebellum with formation of iron complexes is still not clear. Nevertheless, one of the most plausible explanations is a change in BBB permeability. It impedes the influx of toxic molecules from blood into the brain. However, this impairment is controversial $(20,21)$. Higher permeability of BBB renders some substances, toxins and harmful material to leak out of the bloodstream into the brain cells and tissues. Iron from the blood can penetrate through the BBB and accumulate within neurons and glial cells. Iron accumulation, electrondense ferritin granules in the microglia cytoplasm and Bergmann cells of the cerebellar cortex after recurrent extravasation were observed (22). In addition, the occurrence of iron accumulation in the brain can raise neuronal damage with subsequent release of iron sequestered in intracellular structures of neuronal cells. E.g., Salford et al (23) found a significant relationship between the RF EMF exposure and the number of dark neurons what may assume a serious neuronal damage.

Ferritin is the form of an iron storage nonheme-protein with diameter up to $12 \mathrm{~nm}$ consisting of $6 \mathrm{~nm}$ iron core. It has multiphase structure - superparamagnetic ferrihydrite, ferromagnetic magnetite and antiferromagnetic hematite, where all parts are covered with polypeptide. Three iron-related structures were found in the brain: the diamagnetic, paramagnetic and superparamagnetic (24). In addition, ferrimagnetic magnetite or maghemite were also found (25). The external magnetic field may also influence ferrimagnetic iron compounds occurring in the nerve cells and in cell membranes. Consequently, biochemical reactions proceeding in a vicinity of these particles could be influenced by interaction with RF EMF. This could result in a production of reactive oxygen species (ROS) that can damage the membranes. Impairment of polypeptide coat of ferritin causes a release of iron from the core. After two hours of exposure, the ability of ferritin to bind an iron was reduced by $20 \%$ (26). Dadras et al (27) observed in vitro change of Tau protein and tubulin conformation and change of axonal microtubuls alignment as the result of static magnetic field on magnetite nanoparticles. Parallel alignment of these structures is important for physiologic function of axons in propagation of action potential. Biological effect of ferrimagnetic material in brain after the RF EMF exposure may reflect the energy absorption following energy dissipation in the surrounding tissue and cells (28). Nanoparticles of ferritin core increase their internal energy under exposure to EMF. This energy dissipates to the polypeptide coat with change of its molecular structure and function and following iron release. Some studies confirm a harmful bioeffect of RF EMF on molecular level (29-31) and a harmful bioeffect of ferromagnetic nanoparticles. Though, the number, the size, distribution and magnetic properties of nanoparticles is crucial (32). However, the results of these theoretical models and in vitro experiments may not be entirely applicable to human brain. Størmer (33) hypothesized, that the membranes surrounding the human brain (Pia and Dura mater) may play an important role in shielding against external electromagnetic radiation. The time period between the exposure to RF EMF and a sacrifice of animal seems to be important both for penetration through BBB with subsequent accumulation and damage of neuronal cells by iron release. Rabbits in this study were sacrificed 15 minutes after the exposure. This time can outline moderate iron accumulation in neuronal tissue and cells.

\section{Conclusion}

Our experiment revealed structural changes of Purkinje cells and iron and aluminium accumulations in stratum granulosum of rabbit's cerebellum after exposure to RF EMF. These changes can cause exposure to radiation itself and/or iron through its reactive species formation. Iron can leak from blood to cerebellar tissue over damaged blood - brain barrier or pass through iron sequestered loci in intracellular structures of impaired neuronal cells. We explained a moderate iron accumulation as the result of short time between exposure to RF EMF and sacrifice of the animals.

\section{References}

1. Repacholi MH. Health risks from the use of mobile phones. Toxicol Lett 2001; 120: 323-331.

2. Heikkinen P, Kosma VM, Alhonen L et al. Effects of mobile phone radiation on UV-induced skin tumorigenesis in ornithine decarboxylase transgenic and non-transgenic mice. Int J Rad Biol 2003; 79: 221-233.

\section{3. http://monographs.iarc.fr/ENG/Classification/index.php.}

4. Irmak MK, Fadillioglu E, Gulec M et al. Effects of electromagnetic radiation from a cellular telephone on the oxidant and antioxidant levels in rabbits. Cell Biochem Funct 2002; 20: 279-283. 
5. Ithan A, Gurel A, Armutcu F et al. Ginkgo biloba prevents mobile phoneinduced oxidative stress in rat brain. Clin Chim Acta 2004; 340: 153-162.

6. Juutilainen $\mathbf{J}$, Huuskonen $\mathbf{H}$, Komulainen $\mathbf{H}$. Increased resorptions in CBA mice exposed to low-frequency magnetic fields: An attempt to replicate earlier observations. Bioelectromag 1997; 18: 410-417.

7. Jauchem JR. Effects of low-level radio-frequency ( $3 \mathrm{kHz}$ to $300 \mathrm{GHz}$ ) energy on human cardiovascular, reproductive, immune, and other systems: a review of the recent literature. Int J Hyg Environ Health 2008; 211: 1-29.

8. Meral O, Ozgur E, Kismali G, Guler G, Alpay M, Sel T, Seyhan N. GSM-like radiofrequency exposure induces apoptosis via caspase-dependent pathway in infant rabbits. Bratisl Med J 2016; 117 (11): 672 - 676.

9. Schonborn F, Burkhardt M, Kuster N. Differences in energy absorption between heads of adults and children in the near field of sources. Health Phys 1998; 74: 160-168.

10. Maskey D, Pradhan J, Aryal B et al. Chronic 835-MHz radio-frequency exposure to mice hippocampus alters the distribution of calbindin and GFAP immunoreactivity. Brain Res 2010; 1346: 237-246.

11. Sonmez OF, Odaci E, Bas O, Kaplan S. Purkinje cell number decreases in the adult female rat cerebellum following exposure to $900 \mathrm{MHz}$ electromagnetic field. Brain Res 2010; 1356: 95-101.

12. Lebedeva NN, Sulimov AV, Sulimova OP, Korotkovskaya TI, Gailus T. Investigation of brain potentials in sleeping humans exposed to the electromagnetic field of mobile phones. Crit Rev Biomed Eng 2001; 29 : $125-133$.

13. Mausset-Bonnefont AL, de Sèze R., Privat A. Immunohistochemistry as a tool for topographical semi-quantification of neurotransmitters in the brain. Brain Res Protoc 2003; 10: 148-155.

14. Gruenau SP, Oscar KJ, Folker MT, Rapoport SI. Absence of microwave effect on blood-brain barrier permeability to [14C]sucrose in the conscious rat. Exp Neurol 1982; 75 (2): 299-307.

15. Schirmacher A, Winters $\mathbf{S}$, Fischer $\mathbf{S}$ et al. Electromagnetic fields $(1.8 \mathrm{GHz})$ increase the permeability to sucrose of the blood-brain barrier in vitro. Bioelectromag 2000; 21 (5): 338-345.

16. Rouault TA. Iron metabolism in the CNS: implications for neurodegenerative diseases. Nature Reviews Neuroscience 2013; 14: 551-564.

17. Galuščák R, Hazdra P. Dual-Band Loop Feed With Enhanced Performance. Radioengin 2008; 17 (3): 33-37.

18. Rosli Y, Teoh PJ. The effect of low electromagnetic field in the cerebellar layers of mice. J Biol Scie 2009; 9 (6): 601-606.

19. Burlaka A, Selyuk M, Gafurov M et al. Changes in mitochondrial functioning with electromagnetic radiation of ultra high frequency as revealed by electron paramagnetic resonance methods. Inter J Rad Biol. 2014; 90 (5): 357-362.
20. Gruenau SP, Oscar KJ, Folker, Rapaport SI. Absence of microwave effect on blood-brain barrier permeability to $[14 \mathrm{C}]$ sucrose in the conscious rat. Exper Neurol 1982; 75: 299-307.

21. Schirmacher A, Winters S, Fischer $\mathbf{S}$ et al. Electromagnetic fields $(1.8 \mathrm{GHz})$ increase the permeability to sucrose of the blood-brain barrier in vitro. Bioelectromag 2000; 21: 338-345.

22. Koeppen AH, Borke RC. Experimental Superficial Siderosis of the Central Nervous System. I. Morphological Observations. J Neuropathol Exp Neurol 1991; 50 (5): 579-594.

23. Salford LG, Brun AE, Eberhardt JL, Malmgren L, Persson BR. Nerve cell damage in mammalian brain after exposure to microwaves from GSM mobile phones. Environ Health Perspect 2003; 111(7): 881-883.

24. Allen PD, St Pierre TG, Chua-anusorn W, Ström V, Rao KV. Lowfrequency low-field magnetic susceptibility of ferritin and hemosiderin. Biochim Biophys Acta 2000; 1500 (2): 186-196.

25. Kirschvink JL, Kobayashi-Kirschvink A, Woodford BJ. Magnetite biomineralization in the human brain. Proc Natl Acad Sci USA 1992; 89: 7683-7687.

26. Céspedes O, Shoogo U. Radio frequency magnetic field effects on molecular dynamics and iron uptake in cage proteins. Bioelectromag 2010; 31 (4): 311-317.

27. Dadras A, Riazi GH, Afrasiabi A et al. In vitro study on the alterations of brain tubulin structure and assembly affected by magnetite nanoparticles. J Biol Inorg Chem 2013; 18 (3): 357-369

28. Kirschvink, JL. Nonthermal levels of radiation to biological systems. Bioelectromag 1996; 17: 187-194.

29. de Pomerai DI, Smith B, Dawe A et al. Microwave radiation can alter protein conformation without bulk heating. FEBS Lett 2003; 543 (1-3): 93-97.

30. Cranfield C, Wieser HG, Al Madan J, Dobson J. Preliminary evaluation of nanoscale biogenic magnetite-based ferromagnetic transduction mechanisms for mobile phone bioeffects. IEEE Trans Nanobiosci 2003; 2 (1): 40-43.

31. Guney M, Ozguner F, Oral B, Karahan N, Mungan T. $900 \mathrm{MHz}$ radiofrequency-induced histopathologic changes and oxidative stress in rat endometrium: protection by vitamins $\mathrm{E}$ and $\mathrm{C}$. Toxicol Ind Health 2007; 23 (7): 411-420.

32. Dobson J, St. Pierre TG. Theoretical Evaluation of Cellular Phone Safety Aspects. Electromag Biol Med 1998; 17 (3): 351-359.

33. Størmer FC. Magnetite in dura and pia mater in human brain. A shield against electromagnetic radiation? Med Hyp 2014; 82: 122-123.

Received May 11, 2017. Accepted May 30, 2017. 